\title{
Optical visibility range estimation device for safety improvement of unmanned navigation
}

\author{
Denis Nacharov ${ }^{1, *}$ and Yurij Mickhayluck ${ }^{1}$ \\ ${ }^{1}$ Sevastopol State University, Electronics Department, 299053 Sevastopol, Russian Federation
}

\begin{abstract}
Novel methodology for daytime optical visibility range estimation using digital images is presented. Proposed methodology is proved to be effective by results of computational experiments that are presented in this article. Technical suggestions about calibration, hardware and software requirements visibility range estimation device are made.
\end{abstract}

\section{Introduction}

Modern advanced automatic systems, such as unmanned vessels, that are being developed nowadays have to be equipped with systems for autonomous navigation, collection, processing, reception and transmission of information. The effectiveness of performance of unmanned vessel is largely determined by its ability to adapt to changing sailing conditions, which in particular include visibility conditions. Fig.1 shows the block diagram of the onboard subsystem of the remote control system of unmanned vessel, that is being developed in [1]. Image acquisition and processing unit performs visibility range (VR) estimation and objects detection and recognition on video images. Image acquisition and processing unit, as well as other units and systems of an unmanned vessel, has to satisfy a number of requirements for mass, metrological and other indicators.

Known VR estimation devices are characterized either by low accuracy and the need for human participation in the measurement process [2], or by high cost, since they require not only a receiver, but also an optical radiation source [3].

Novel methodology for estimation the daytime optical VR using digital images is presented in this article. The results of a computational experiments of VR estimation are presented. The requirements for the hardware and software of the daytime optical VR estimation device that implements the proposed methodology are analysed.

\section{Visibility range estimation using digital images}

The daytime optical VR $D_{v}$ is defined as the greatest distance at which a black object is visible in the daytime against the sky, if the angular dimensions of the object are not less than 15 '[4]. The basis of the VR estimation technique is the model [5], according to which the brightness of the image recorded in a cloudy atmosphere can be represented by the following expression

$$
I_{i}=R_{i} e^{-\beta d}+\left(1-e^{-\beta d}{ }_{i}\right) L_{\infty}
$$

\footnotetext{
${ }^{*}$ Corresponding author: d.v.nacharov@sevsu.ru
} 
where $R_{i}$ - brightness of the $i^{\text {th }}$ pixel of the image, determined by the reflectivity of the scene object; $d_{i}$ - distance from the recording device to the scene object; $L_{\infty}$ - the brightness of the sky near the horizon [4].

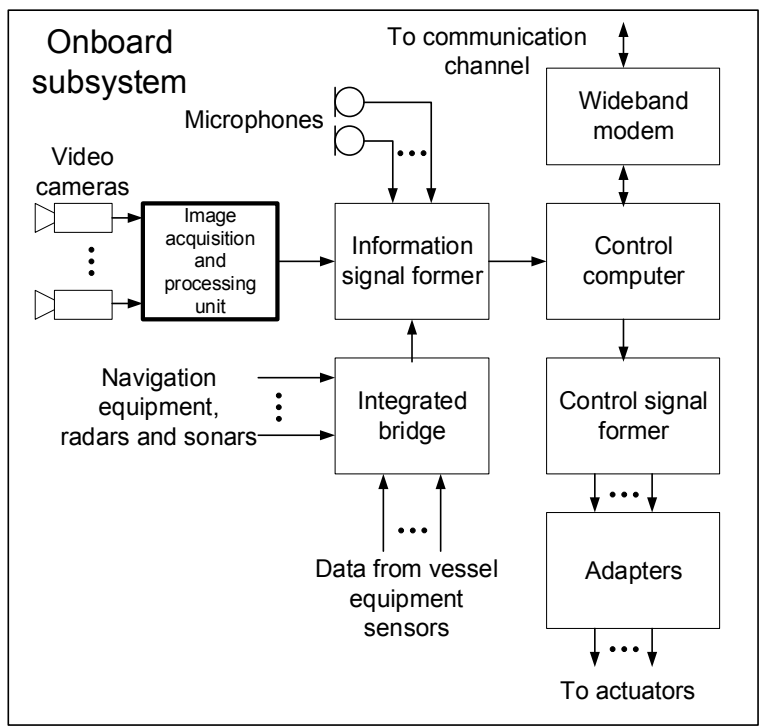

Fig. 1. Block diagram of the onboard subsystem of the remote control system of unmanned vessel.

When observing a black object $(R=0)$, we obtain

$$
I_{\text {black }}=\left(1-e_{\text {black }}^{-\beta d}\right) L_{\infty}
$$

where $I_{\text {black }}$ - pixel brightness of a black object in the image; $d_{\text {black }}$ - distance from the recording device to the black object.

The contrast $K_{\text {black }}$ between brightness $I_{\text {black }}$ and brightness $L_{\infty}$ is

$$
K_{\text {black }}=\frac{L_{\infty}-I_{\text {black }}}{L_{\infty}}=\frac{L_{\infty}-\left(1-e^{-\beta d_{\text {black }}}\right) L_{\infty}}{L_{\infty}}=e^{-\beta d_{\text {black }}} .
$$

Thus, the daily optical VR is the distance at which the contrast will be equal to the threshold contrast. In assessing the VR, the average threshold contrast value for daytime conditions is 0.02 [4].

Performing the substitution in the expression (3) and expressing $D_{v}$, we obtain

$$
D_{v}=-\frac{\ln 0,02}{\beta}=\frac{3,91}{\beta} \text {. }
$$

Thus, the problem of evaluating the VR could be solved by determining the attenuation coefficient $\beta$ and subsequent calculation of $D_{v}$ using expression (4).

In the presence of a pair of images (recorded in a clear and cloudy atmosphere) for a known distance from the scene object, there are two unknown values in expression (1) $L_{\infty}$ and $\beta$. It can also be determined by writing down the expressions for the two brightness values of the image corresponding to the presence of an aerosol, and solving the system of equations

$$
\left\{\begin{array}{l}
I_{1}=R_{1} e^{-\beta d_{1}}+\left(1-e^{-\beta d_{1}}\right) L_{\infty} \\
I_{2}=R_{2} e^{-\beta d_{2}}+\left(1-e^{-\beta d_{2}}\right) L_{\infty} .
\end{array}\right.
$$

Equations (5) is a system of nonlinear equations, the solution of which can be found numerically. To determine the conditions for solving the system of equations (5), we 
consider the plan of the VR estimation system, shown in Fig. 2. The brightnesses $R_{1}$ and $R_{2}$ can be determined using an object with known brightnesses measured in a clear atmosphere (when $\beta=0$ ) as a marker.

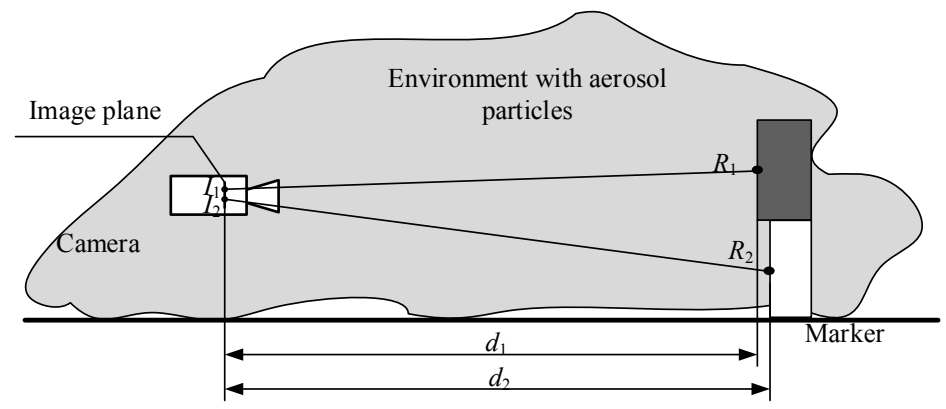

Fig. 2. Daytime visibility range estimation system by using digital images.

To find the dependence of the relative error in the estimation of the VR on the value of the VR, let's define the differential $\Delta D_{v}$ using expression

$$
\Delta D_{v}=\left(-\frac{\ln 0,02}{\beta}\right)^{\prime} \Delta \beta=\frac{-\ln 0,02}{\beta^{2}} \Delta \beta .
$$

To determine $\Delta \beta$, we consider the case of equality of distances to marker points. Then, with $d_{1}=d_{2}$ system (5) could be written as

$$
\left\{\begin{array}{l}
I_{1}=R_{1} t+(1-t) L_{\infty} \\
I_{2}=R_{2} t+(1-t) L_{\infty}
\end{array}\right.
$$

where $t=e^{-\beta d_{1}}$.

Having expressed $L_{\infty}$ and performing the substitution, we obtain

$$
I_{1}=R_{1} t+(1-t) \frac{I_{2}-R_{2} t}{1-t} ; t=\frac{I_{1}-I_{2}}{R_{1}-R_{2}} .
$$

Since $\beta=\frac{-\ln t}{d_{1}}$ and $\Delta \beta=\frac{\Delta t}{t d_{1}}$, we get

$$
\Delta D_{v}=\frac{-\ln 0,02}{\beta^{2}} \frac{\Delta t}{t d_{1}}
$$

And then $\Delta t$

$$
\Delta t=\frac{\Delta I_{1}-\Delta I_{2}}{R_{1}-R_{2}}=\frac{\Delta I}{R_{1}-R_{2}} .
$$

Thus, the error in the estimation of the VR is basically determined by the errors in the measurement of brightness $I_{1}$ and $I_{2}$, and also depends on the difference $\left(R_{1}-R_{2}\right)$ that determines the contrast of the marker. With an 8-bit representation of the brightness of the digital image, we take a value $\Delta I$ to be equal to 1 .

As a result of substitutions $\beta, t$ and $\Delta t$ in expression (7), the analytical dependence $\Delta D_{\text {vrel }}\left(D_{v}\right)$ of the relative error of the VR estimate (in \%) on the value of the VR has the following form

$$
\Delta D_{v r e l}\left(D_{v}\right)=\frac{\Delta D_{v}}{D_{v}}\left(D_{v}\right)=\frac{D_{v} \frac{1}{R_{1}-R_{2}}}{-\ln 0,02 \cdot d_{1} e^{\frac{d_{1} \ln 0,02}{D_{v}}}} \cdot 100 \%
$$


Fig. 3 shows the graphs of dependences (9) for various values of the distances $d_{1}$ when $\left(R_{1}-R_{2}\right)=255$. Analyzing graphs of Fig. 3 it can be concluded that the boundaries of the range of estimated VRs depend on the distance to the marker.

Fig. 4 shows the analytical and experimental dependences $\Delta D_{\text {vrel }}\left(D_{v}\right)$ for the same marker. To calculate the experimental dependence, a set of images was synthesized [6] with values of $\beta$ ranging from 0.0005 to $0.1 \mathrm{~m}^{-1}$, which corresponds to a change in VR from $39.1 \mathrm{~m}$ up to $7820 \mathrm{~m}$.

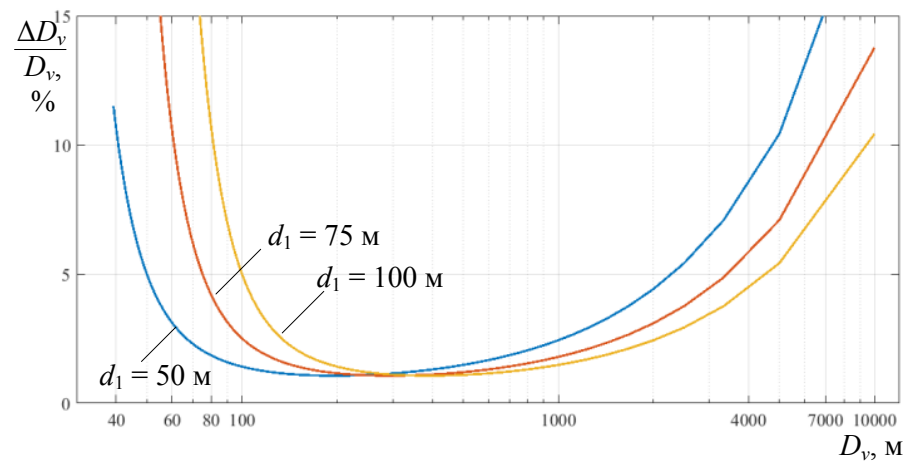

Fig. 3. Dependencies $\Delta D_{v r e l}\left(D_{v}\right)$ with distance $d_{1}$ used as a parameter.

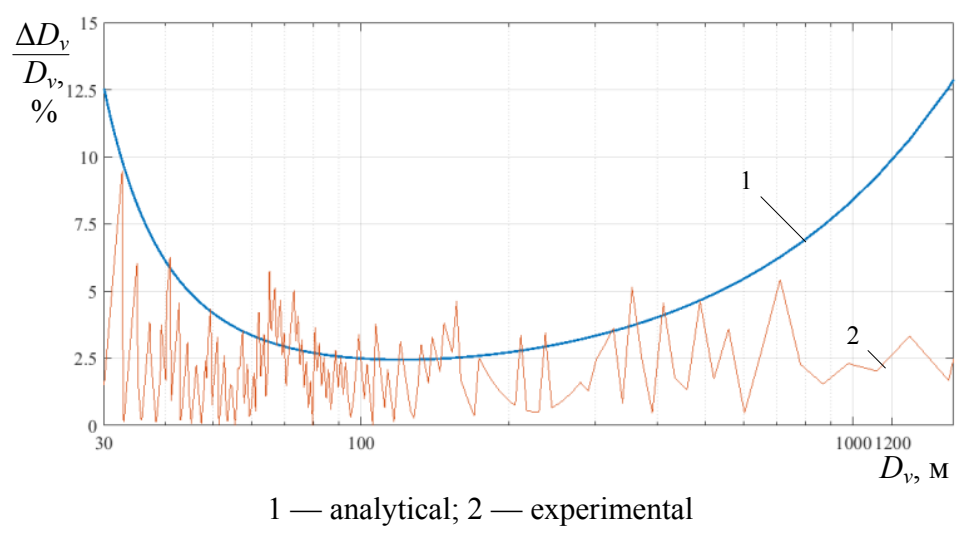

Fig. 4. Analytical and experimental dependences of accuracy as a function of visibility.

The obtained results allow us to justify the requirements for the object used as a marker:

1) to reduce the relative error of the VR estimation, the difference in the brightness of the marker $\left(R_{1}-R_{2}\right)$ should be chosen as the largest - equal to 255 for an 8-bit representation of brightness;

2) the distance to the marker $d_{1}$ should be selected concerning the min and max values VR to be estimated, providing range of values for which the relative error does not exceed the specified value.

When deploying a VR estimation system at a real object, for example, a ship, structural elements located at a known distance from the recording device can be used as markers. Mandatory conditions - the marker must be stationary relative to the object on which the system is installed, and its illumination should be determined by natural light sources.

To clarify the brightness of the marker, it is proposed to calibrate, as a result of which the magnitude of the indicator is determined in an atmosphere close to clear. Fig. 5 shows a calibration plan for the VR estimation system. Calibration involves the use of a black object 
located at a known distance from the recording device, which allows us to describe the recorded brightness $I_{\text {black }}$ by expression (2). From (2) we get

$$
\beta_{c}=-\frac{1}{d_{\text {black }}} \ln \left(\frac{L_{\infty}-I_{\text {black }}}{L_{\infty}}\right)
$$

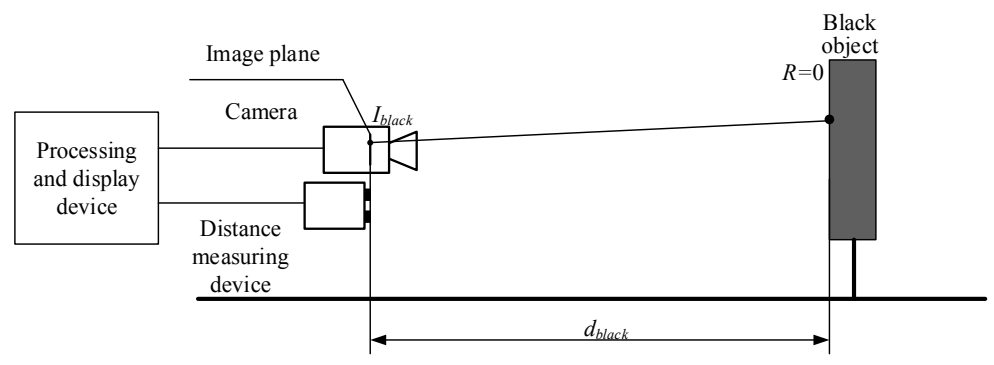

Fig. 5. Calibration of visibility range estimation system.

The actual brightness of the sky near the horizon, measured during calibration, can be used as a value of $L_{\infty}$. Values $R_{1}, R_{2}$ are determined from expressions

$$
R_{1}=\frac{I_{1 c}-\left(1-e^{-\beta_{c} d_{1}}\right) L_{\infty}}{e^{-\beta_{c} d_{1}}} ; \quad R_{2}=\frac{I_{2 c}-\left(1-e^{-\beta_{c} d_{2}}\right) L_{\infty}}{e^{-\beta_{c} d_{2}}},
$$

where $I_{1 c}, I_{2 c}$ - the brightness values of the markers measured during calibration.

Thus, a single calibration in arbitrary observation conditions allows us to specify the brightness values $R_{1}, R_{2}$ of the marker and to ensure the accuracy of the VR estimation using the proposed method will match the result of the model experiment.

\section{Conclusions}

The methodology for estimating the daytime optical VR using digital images was developed. The relative error in the estimation of the VR is analysed, the requirements for the object used as a marker are substantiated. Equations for determining the boundaries of the range of the VR estimation are obtained. A calibrating procedure is proposed.

Studies were supported by the Ministry of Science and Higher Education the Russian Federation (project RFMEFI57817X0259).

\section{References}

1. Nacharov D., Mickhayluck Y., Iskiv V. Objects Discernibility Improvement as Part of Unmanned Vessel Control System // Proc. of 2018 IEEE East-West Design and Test Symposium, EWDTS. P.475-479 (2018)

2. Sharonov V.V. Nabludenie I vidimost [Surveillance and visibility]. Moscow, USSR Academy of Sciences Publ. 80 p. (In Russian) (1943)

3. Vaisala Transmissometer LT31 // VAISALA. URL: http://www.vaisala.com/en/ products/visibilitysensors/Pages/LT31.aspx/ (date of request 15.06.2019)

4. Nacharov D. V., Mickhayluck Y.P. [The system of visibility range estimation using digital television image as an instrument of transport application efficiency upgrading]. Sbornik nauchnykh trydov AVMS im. Nakhimova - Collection of scientific works of AVMS im. Nakhimova, Vol. 10, No. 2, pp. 121-125. (In Russian) (2012) 
5. Kovalev V.A. Vidimost $\mathrm{v}$ atmosphere I ee opredelenie [Visibility in atmosphere and its measurements]. Leningrad. Gidrometizdat Publ. 215 p. (In Russian) (1988)

6. Nayar S.K. Vision in Bad Weather / S.K. Nayar, S.G. Narasimhan // Proc. of the 7th IEEE International Conf. on Computer Vision. Vol. 2. P.820-827 (1999) 